\title{
A COMPARISON BETWEEN MILP AND MINLP APPROACHES TO OPTIMAL SOLUTION OF NONLINEAR DISCRETE TRANSPORTATION PROBLEM
}

\author{
Uroš Klanšek \\ Faculty of Civil Engineering, University of Maribor, Slovenia
}

Submitted 24 February 2014; resubmitted 16 May 2014; accepted 26 May 2014; first published online 10 July 2014

\begin{abstract}
Finding an exact optimal solution of the Nonlinear Discrete Transportation Problem (NDTP) represents a challenging task in transportation science. Development of an adequate model formulation and selection of an appropriate optimization method are thus significant for attaining valuable solution of the NDTP. When nonlinearities appear within the criterion of optimization, the NDTP can be formulated directly as a Mixed-Integer Nonlinear Programming (MINLP) task or it can be linearized and converted into a Mixed-Integer Linear Programming (MILP) problem. This paper presents a comparison between MILP and MINLP approaches to exact optimal solution of the NDTP. The comparison is based on obtained results of experiments executed on a set of reference test problems. The paper discusses advantages and limitations of both optimization approaches.

Keywords: transportation problems; discrete transporting flows; nonlinear costs; optimization methods; mixed-integer linear programming; mixed-integer nonlinear programming.
\end{abstract}

\section{Notations}

Indices:

$i \quad$ - index of suppliers, $i \in I$;

$j \quad$ - index of demanders, $j \in J$;

$k \quad-$ index of alternative discrete solutions for transporting flows, $k \in K(i, j)$.

Constants:

$c_{i, j} \quad$ - cost parameters;

$d_{j} \quad$ - demands at destinations;

$f_{i, j, k} \quad$ - cost function values of alternative discrete solutions for transporting flows;

$P A \quad$ - curve fitting parameter for cost function $A\left(x_{i, j}\right)$;

$P B$ - curve fitting parameter for cost function $B\left(x_{i, j}\right)$;

$q_{k} \quad$ - alternative discrete solutions for transporting flows;

$s_{i} \quad$ - supply capacities of sources;

$S$ - input parameter for cost functions $A\left(x_{i, j}\right)$,

Variables: $B\left(x_{i, j}\right), E\left(x_{i, j}\right)$ and $F\left(x_{i, j}\right)$.

$A\left(x_{i, j}\right)$ - nonlinear continuous arc-tangent approximation of a five-step piece-wise linear cost function;

$B\left(x_{i, j}\right)$ - nonlinear continuous arc-tangent approximation of a piece-wise linear cost function with three gradients;
Cost - total cost of single cargo transportation;

$C\left(x_{i, j}\right)$ - regular quadratic cost function;

$D\left(x_{i, j}\right)$ - square root cost function;

$E\left(x_{i, j}\right)$ - continuous nonconvex cost function with peak;

$f_{i, j}\left(x_{i, j}\right)$ - cost functions of transporting flows;

$F\left(x_{i, j}\right)$ - non-convex function with multiple valleys and peaks;

$x_{i, j} \quad$ - transporting flows;

$y_{i, j, k} \quad$ - binary decision variables for the selection of optimal discrete transporting flows.

\section{Introduction}

Transportation Problem (TP) generally presents a network-flow optimization problem. The basic aim of the $\mathrm{TP}$ is to achieve the minimum total transportation cost for a shipment of single merchandise from a number of suppliers to a number of demanders without exceeding capacities of suppliers and by fulfilling requirements of demanders. From the viewpoint of the optimization problem formulation, the transportation costs, supply and demand quantities usually represent input parameters while transporting flows denote decision variables.

Traditionally, the unit costs of commodity transportation from suppliers to demanders are assumed to have constant values within the TP formulation. In this way, the total transportation cost objective function 
takes linear form and the TP can be efficiently solved applying Linear Programming (LP) approach. However, in real operations, the unit cost of transporting cargo often decreases as the amount of cargo increases, mostly on account of economy of scale. For this reason, the dependence between the amount of cargo and the unit cost of its transportation was extensively discussed in published literature.

For instance, Dangalchev (1996) approximated relationships between quantities and unit costs of transportation employing partially-linear functions in the TP model. Yan and Luo (1999) demonstrated that the unit costs for transportation of goods from suppliers to demanders can be formulated in the TP model applying linear fixed charge cost functions. Still, the dependence between the amount of commodity and the transportation cost can be also, in many cases, suitably approximated using nonlinear terms, such as quadratic, square-root or power functions among others, see e.g. Mizutani and Yamashita (2013). As soon as the cost of transportation becomes nonlinearly dependent on the discrete amount of cargo units transported, the TP turns into the nonlinear discrete network-flow problem.

Different variations of Nonlinear Discrete Transportation Problem (NDTP) can be widely found in many industrial fields including logistics, production, engineering, management, etc. However, finding an exact optimal solution of the NDTP still represents a most challenging task. Classical nonlinear techniques for constrained optimization can be applied to solve nonlinear transportation problems which contain integer variables by rounding real-valued points to nearest integers. Though, the application of such techniques may sometimes lead to sub-optimal results and be less appropriate for achieving high quality exact discrete solutions in cases of large-scale problems.

NDTPs that occur in practice often represent NPhard problems of combinatorial optimization. In this way, the development of an appropriate model formulation and the selection of an adequate optimization method are significant for finding valuable solution of the NDTP. When nonlinearities appear in the objective function, a specific NDTP can be set directly as a MixedInteger Nonlinear Programming (MINLP) task, see e.g. Ozsen et al. (2009); Monteiro et al. (2010); Romeijn and Sargut (2011); Ağralı et al. (2012); Carrizosa et al. (2012). On the other hand, the nonlinear transportation cost can be also approximated into a linear form (see e.g. Kameshwaran and Narahari (2009); Madadi et al. (2010); Christensen et al. (2013)) and the NDTP can be thus reformulated into a Mixed-Integer Linear Programming (MILP) problem.

The main purpose of this paper is to present a comparison between MILP and MINLP approaches to exact optimal solution of the NDTP. The implemented research presents a natural continuation of the work introduced in reference (Klanšek 2014) where the performance of different exact MINLP optimization methods, i.e. the Extended Cutting Plane (ECP) method by Westerlund and Pettersson (1995), the Branch and Re- duce (BR) method by Ryoo and Sahinidis (1996), the Augmented Penalty/Outer-Approximation/EqualityRelaxation method (AP/OA/ER) by Viswanathan and Grossmann (1990), the Branch and Cut (BC) method by Lin and Schrage (2009), and the Simple Branch and Bound (SBB) method by Leyffer (2001), was tested on a set of reference NDTPs.

In a view of precedent work, the original NDTP formulation was linearized and converted into a MILP problem. Afterwards, the developed MILP model was applied to solve the same reference test problems from the previous research (Klanšek 2014). MILP solutions of test problems were obtained by CPLEX algorithm (GAMS/CPLEX 12.0 User Notes 2012), an implementation based on branch and bound (BB) method (Land, Doig 1960), and compared with those achieved by MINLP methods. The paper discusses attained results of performed tests as well as MILP and MINLP modelling capabilities. The comparison shows advantages and limitations of both optimization approaches.

\section{General Optimization Problem Formulations}

The NDTP represents the nonlinear discrete problem of combinatorial optimization. In this way, the NDTP can be directly formulated as a problem of MINLP optimization. The MINLP optimization problem can be generally set as:

$$
\begin{aligned}
& \text { Minimize } z=f(\boldsymbol{x})+\boldsymbol{d}^{\mathrm{T}} \boldsymbol{y}, \\
& \text { subject to: } \\
& \boldsymbol{h}(\boldsymbol{x})=\mathbf{0} ; \\
& \boldsymbol{g}(\boldsymbol{x}) \leq \mathbf{0} ; \\
& \boldsymbol{A} \boldsymbol{x}+\boldsymbol{B} \boldsymbol{y} \leq \boldsymbol{b} ; \\
& \boldsymbol{x} \in X=\left\{\boldsymbol{x} \in R^{n}: \boldsymbol{x}^{\mathrm{LO}} \leq \boldsymbol{x} \leq \boldsymbol{x}^{\mathrm{UP}}\right\} ; \\
& \boldsymbol{y} \in Y=\{0,1\}^{m},
\end{aligned}
$$

(MINLP-G)

where: $x$ is the vector of continuous variables defined in compact set $X ; \boldsymbol{y}$ is the vector of binary decision variables; $\boldsymbol{d}^{\mathrm{T}}$ is the vector of constants. Continuous variables $\boldsymbol{x}$ can be set linearly or nonlinearly in the objective function and constraints while binary variables $y$ can only occur in linear terms. Functions $f(\boldsymbol{x}), \boldsymbol{h}(\boldsymbol{x})$ and $\boldsymbol{g}(\boldsymbol{x})$ denote nonlinear functions comprised in the objective function $z$, equality and inequality conditions, respectively. Thereafter, the expression $\boldsymbol{A x}+\boldsymbol{B} \boldsymbol{y} \leq \boldsymbol{b}$ introduces a subset of mixed linear (in)equality constraints. At this point, all functions $f(\boldsymbol{x}), \boldsymbol{h}(\boldsymbol{x})$ and $\boldsymbol{g}(\boldsymbol{x})$ have to be continuous and differentiable.

As soon as the nonlinear expressions are appropriately linearized, the MINLP task can be reformulated into the MILP problem. The general formulation of the MILP problem can be presented using following expressions:

Minimize $z=\boldsymbol{c}^{\mathrm{T}} \boldsymbol{x}+\boldsymbol{d}^{\mathrm{T}} \boldsymbol{y}$

subject to:

$$
\begin{aligned}
& \boldsymbol{A} \boldsymbol{x}+\boldsymbol{B} \boldsymbol{y} \leq \boldsymbol{b} ; \\
& \boldsymbol{x} \in X=\left\{\boldsymbol{x} \in R^{n}: \boldsymbol{x}^{\mathrm{LO}} \leq \boldsymbol{x} \leq \boldsymbol{x}^{\mathrm{UP}}\right\} \\
& \boldsymbol{y} \in Y=\{0,1\}^{m} .
\end{aligned}
$$

Similarly as the MINLP-G optimization problem, also the MILP-G one includes the objective function 
which is subjected to various (in)equality conditions with continuous and binary decision variables. Though, when the problem of MILP optimization is taken into consideration, both types of decision variables can appear only linearly in its formulation.

Thus, the nonlinear expressions of the objective function $f(x)$ must be linearized into the form $\boldsymbol{c}^{\mathrm{T}} \boldsymbol{x}$ and the nonlinear (in)equality constraints $\boldsymbol{h}(\boldsymbol{x})$ and $\boldsymbol{g}(\boldsymbol{x})$ have to be approximated using linear terms and set in the formulation as mixed linear restrictions $\boldsymbol{A x}+\boldsymbol{B} \boldsymbol{y} \leq \boldsymbol{b}$. Nonlinear terms can be set into a linear form using discretized or (piece-wise) linear functions. Note, however, that perfect matching between the nonlinear continuous function and the discretized one can be reached in discrete points while the (piece-wise) linear approximation of nonlinear expression can only be achieved under a certain linearization error.

\section{Optimization Model Formulations}

Compared with previously introduced general optimization problem formulations, the NDTP model contains more specific objective function, decision variables and (in)equality conditions. For instance, the total cost of single cargo transportation from suppliers to demanders can be defined as the objective function of the NDTP model using the following form:

$$
\text { Cost }=\sum_{i \in I} \sum_{j \in J} f_{i, j}\left(x_{i, j}\right) \text {, }
$$

where: expressions $f_{i, j}\left(x_{i, j}\right)$ are applied to comprehend cost functions of transporting flows $x_{i, j}$ from suppliers $i, i \in I$, to demanders $j, j \in J$, within the criterion of optimization.

Since functions $f_{i, j}\left(x_{i, j}\right)$ generally represent nonlinear terms, the NDTP model that includes the objective function formulation as given in Eq. (1) can be exactly solved to optimality only by MINLP approach. However, the nonlinear objective function can be discretized and formulated as linear part of the optimization model employing the following equation:

$$
\text { Cost }=\sum_{i \in I} \sum_{j \in J} \sum_{k \in K(i, j)} f_{i, j, k} y_{i, j, k},
$$

where: $f_{i, j, k}$ represents the cost function values of alternative discrete solutions $k, k \in K(i, j)$, for transporting flows $x_{i, j} ; y_{i, j, k}$ denotes binary decision variables which are used to perform the selection of optimal discrete values for continuous variables $x_{i, j}$ from the developed superstructure of alternatives.

The following equality condition should be satisfied in order to establish optimal discrete solutions for transporting flows $x_{i, j}$ out of the generated superstructure of alternatives:

$$
\sum_{k \in K(i, j)} y_{i, j, k} q_{k}=x_{i, j}, i \in I ; j \in J
$$

where: discrete constants $q_{k}$ are defined as discrete solution alternatives for transporting flows $x_{i, j}$. The discrete constant option $q_{k}$ is chosen as the discrete solution for the continuous variable $x_{i, j}$ as soon as the obtained value of the allocated binary variable $y_{i, j, k}$ is equal to 1 . Otherwise, when the gained value of binary variable $y_{i, j, k}$ is equal to 0 , the discrete constant option $q_{k}$ is rejected.

For the purpose of assuring that exactly one discrete value $q_{k}$ is selected as the discrete optimal solution for each continuous variable $x_{i, j}$, the following logical constraint should be fulfilled:

$$
\sum_{k \in K(i, j)} y_{i, j, k}=1, i \in I ; j \in J \text {. }
$$

The total outgoing shipment of cargo from each stated source $i, i \in I$, cannot exceed its available supply capacity. Hence, the supply limitation has to be included into the optimization model as:

$$
\sum_{j \in J} x_{i, j} \leq s_{i}, i \in I
$$

where: $s_{i}$ represents available supply capacities of incorporated sources.

Similarly, the total incoming shipments of cargo to each considered destination $j, j \in J$, are required to satisfy its total demand. In this way, the following demand condition has to be comprehended in the optimization model:

$$
\sum_{i \in I} x_{i, j} \geq d_{j}, j \in J
$$

where: $d_{j}$ denotes demands at comprised destinations. Note here that the solvability of handled optimization problem is ensured only when the total supply exceeds the total demand, i.e. when:

$$
\sum_{i \in I} s_{i} \geq \sum_{j \in J} d_{j}, i \in I ; j \in J .
$$

As regards solution quality, the widest feasible space for transporting flows can be determined as $0 \leq x_{i, j} \leq$ $\min \left\{s_{i}, d_{j}\right\}$. However, in integer optimization, the transporting flows $x_{i, j}$ may take only those discrete values $q_{k}$ which were inserted into the superstructure of discrete solution alternatives. Accordingly, the superstructure of discrete alternatives should be generated attentively so that quality solution can be achieved.

The NDTP can be formulated as a MINLP optimization problem taking into account exact nonlinear shape of the objective function, as given in Eq. (1), and the set of linear conditions as presented in Eqs (3-7). If the exact nonlinear objective function is replaced by discretized one, as defined in Eq. (2), the same problem can be also solved by MILP optimization.

\section{Optimization Methods}

The exact solution methods for (non)linear discrete optimization problems require considerably more mathematical computation than those for similarly sized (non) linear continuous programs. Therefore, even simply formulated integer programming models may require large amount of process time to be solved to optimality on account of their combinatorial size. The computational 
intensity of the optimization search in integer programming often demands significant amount of physical memory.

Despite its ease of formulation, the NDTP belongs to the class of combinatorial problems that are difficult to be optimally solved. In this way, the comparisons between different modelling approaches and optimization methods may provide useful information about which one of them is suitable for solving the stated problem.

In previous research (Klanšek 2014), the performance of five different state-of-the-art MINLP optimization methods was tested on a set of reference NDTPs. The evaluated MINLP methods included the ECP method (Westerlund, Pettersson 1995) of the AlphaECP software (Westerlund, Pörn 2002), the BR method (Ryoo, Sahinidis 1996) of the BARON software (Sahinidis, Tawarmalani 2008), the AP/OA/ER method of the DICOPT software (Viswanathan, Grossmann 1990), the BC method of the LINDOGlobal software (Lin, Schrage 2009), and the SBB method of the same designated software (Leyffer 2001).

The most performed tests revealed that each MINLP method was able to solve a specific NDTP to a better solution than the other considered MINLP techniques. Such differences between obtained results were expected to a certain degree since the applied test problems represented combinatorial and, in most of cases, nonconvex MINLP problems for which the global optimal solution is generally difficult to be reached.

In this paper, the MILP model for the NDTP, formulated using Eqs (2-7), was applied to solve the same reference test problems for the purpose of comparison between both optimization approaches. The MILP search for the optimal solutions of test problems was performed by CPLEX algorithm (GAMS/CPLEX 12.0 User Notes 2012), an implementation based on BB method (Land, Doig 1960).

The BB optimization procedure manages a search tree comprised of nodes. Each node represents an LP subproblem to be solved, checked for integrality, and possibly analysed further. During the search process, the $\mathrm{BB}$ algorithm solves a series of LP subproblems to compute linear constraints that cut off potential solutions that violate the discreteness conditions. The basic aim of adding linear constraints is to reduce the size of solution domain for continuous LP subproblems represented at the nodes while not removing legal integer solutions. The convergence of the BB optimization algorithm is achieved as soon as all active nodes have been processed within the search tree, i.e. solved or fathomed.

\section{Test Problems}

\subsection{Input Data}

The optimization tests were executed on $7 \times 7$ and $10 \times 10$ node reference problems (Michalewicz et al. 1991). The originally proposed continuous test problems were modified to comprehend only discrete solutions as performed in reference (Klanšek 2014). For both type of test problems, the input data matrices with cost parameters $c_{i, j}$, capacities of sources $s_{i}$ and demands of destinations $d_{j}$ are given in Appendix A.1.

The $7 \times 7$ cost matrix is a symmetrical matrix with zero cost coefficients on the diagonal and six cost parameters with markedly large value of 1000 . The $10 \times 10$ cost matrix contains randomly arranged values of parameters without prominent extremes. Applied cost matrices define the set of balanced test problems since the total supply of sources is, in both cases, equal to the total demand of destinations.

The objective function was set for each test case as:

$$
\text { Cost }=\sum_{i \in I} \sum_{j \in J} \sum_{k \in K(i, j)} c_{i, j} f_{k} y_{i, j, k},
$$

where: $f_{k}$ denotes the cost function values of alternative discrete solutions $q_{k}$ for transporting flows $x_{i, j}$. The cost function values were generated on basis of six different discretized nonlinear cost functions, labelled from $A\left(x_{i, j}\right)$ to $F\left(x_{i, j}\right)$, which were found in reference (Michalewicz et al. 1991), see Appendix A.2.

During generation procedure, input parameters $P A$, $P B$ and $S$ were considered for the purpose of calculating cost function values of alternative discrete solutions. Both values of input parameters $P A$ and $P B$ were set at 1000. A value of 2 was set for input parameter $S$ in the $7 \times 7$ test problems, while in the $10 \times 10$ ones a value of 5 was used.

Cost function $A\left(x_{i, j}\right)$ defines a nonlinear continuous arc-tangent approximation of a five-step piece-wise linear function. The values $f_{k}$ of cost function $A\left(x_{i, j}\right)$, at optional discrete solutions $q_{k}$ for transporting flows $x_{i, j}$, were included into the superstructure of alternatives as shown in Fig. 1.

The formulation of cost function $B\left(x_{i, j}\right)$ determines a nonlinear continuous arc-tangent approximation of a piece-wise linear function with three gradients. Fig. 2 presents the discrete values $f_{k}$ of cost function $B\left(x_{i, j}\right)$ as they were comprehended within the MILP optimization model.

Cost function $C\left(x_{i, j}\right)$ was established by a regular quadratic expression while cost function $D\left(x_{i, j}\right)$ was defined by a square root term. Their cost values $f_{k}$ were set into the optimization model within the superstructure of discrete alternatives as demonstrated in Figs 3 and 4, respectively.

Nonconvex cost functions with peaks and valleys are less often applied in practice. However, such cost functions are frequently used to provide difficult tests for evaluating the solution efficiency of optimization techniques. Michalewicz et al. (1991) proposed two different nonconvex cost functions to be applied in severe performance tests of optimization methods, i.e. cost functions $E\left(x_{i, j}\right)$ and $F\left(x_{i, j}\right)$.

Cost function $E\left(x_{i, j}\right)$ formulates a continuous nonconvex function with peak. Fig. 5 demonstrates the values $f_{k}$ of cost function $E\left(x_{i, j}\right)$ at alternative discrete transportation flows which were incorporated into the MILP optimization model.

The expression of cost function $F\left(x_{i, j}\right)$ creates a highly non-convex diagram with multiple valleys and 


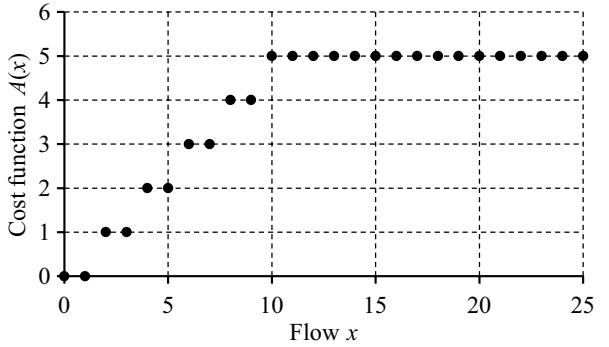

Fig. 1. Discretized cost function $A\left(x_{i, j}\right)$

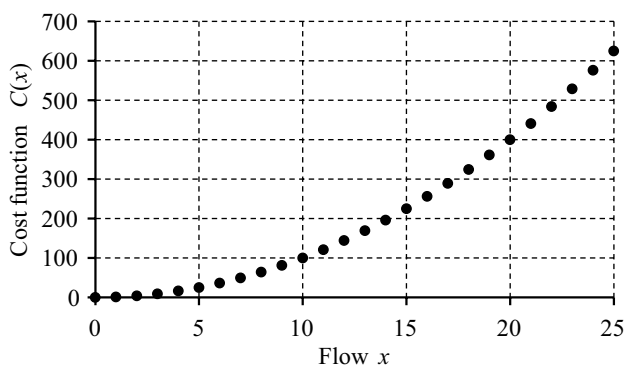

Fig. 3. Discretized cost function $C\left(x_{i, j}\right)$

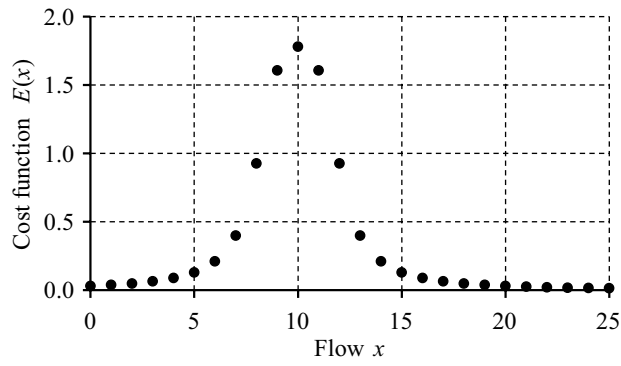

Fig. 5. Discretized cost function $E\left(x_{i, j}\right)$

peaks. Taking into account the form of function $F\left(x_{i, j}\right)$, the superstructure of discrete alternatives comprised cost function values $f_{k}$ as demonstrated in Fig. 6. Fig. 6 shows that the most scattered superstructure of discrete alternatives for cost function values $f_{k}$ was generated on the basis of term $F\left(x_{i, j}\right)$.

\subsection{Optimization Setup}

After generating the superstructure of discrete solution alternatives, a twelve different test problems were determined to be solved to optimality by BB approach, namely $7 \times 7$ and $10 \times 10$ test problems with cost values of alternative discrete transporting flows based on six different nonlinear expressions: $A\left(x_{i, j}\right), B\left(x_{i, j}\right), C\left(x_{i, j}\right)$, $D\left(x_{i, j}\right), E\left(x_{i, j}\right)$ and $F\left(x_{i, j}\right)$, see Appendices A.1 and A.2. Hereinafter, the said test problems will be denoted as test problems $7 \times 7 \mathrm{~A}, 7 \times 7 \mathrm{~B}$ and further up to $7 \times 7 \mathrm{~F}$ as well as $10 \times 10 \mathrm{~A}, 10 \times 10 \mathrm{~B}$ and so on up to $10 \times 10 \mathrm{~F}$, respectively.

Following the structure of input data, the logical constraints (3-4), the supply constraints (5), the demand constraints (6), the condition of supply sufficiency (7) and the objective function (8) were included into the MILP optimization model to obtain optimal solutions for test problems. A high-level language for mathematical programming GAMS (General Algebraic Modelling

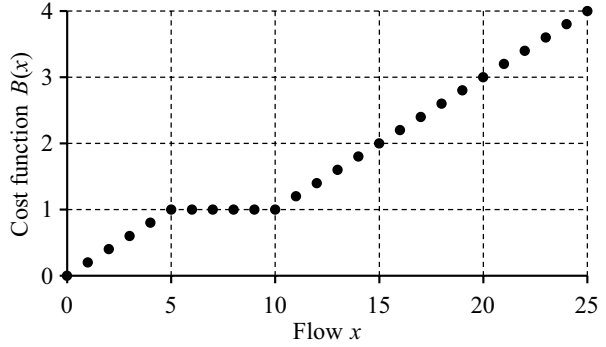

Fig. 2. Discretized cost function $B\left(x_{i, j}\right)$

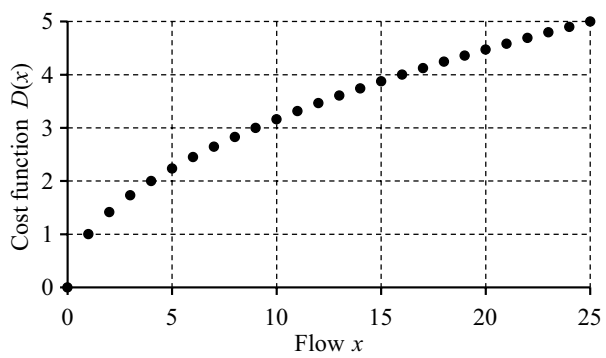

Fig. 4. Discretized cost function $D\left(x_{i, j}\right)$

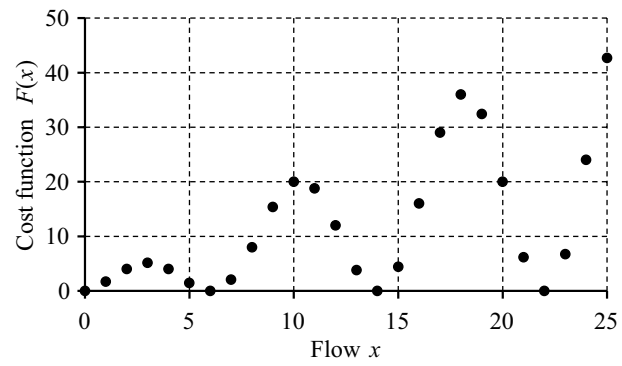

Fig. 6. Discretized cost function $F\left(x_{i, j}\right)$

System) by Brooke et al. (2012) was applied for computer modelling and for data inputs/outputs. The stated test problems were modelled and solved on a 64-bit operating system using the personal computer: Intel Core i7, $2.93 \mathrm{GHz}, 8 \mathrm{~GB}$ RAM and 1 TB hard disc.

The MILP optimization model for $7 \times 7$ test problems comprehended 49 continuous variables, 1087 binary decision variables and 112 constraints while the one for $10 \times 10$ test problems included 100 continuous variables, 616 binary decision variables and 220 constraints. The initial points to start the BB based search for optimal solutions of test problems were generated using classical north-west corner approach.

\subsection{Computational Results}

The convergence of the BB optimization algorithm was achieved under default CPLEX settings. Tables 1 and 2 present the obtained optimal discrete solutions for test problems $7 \times 7 \mathrm{~A}$ and $10 \times 10 \mathrm{~A}$, respectively.

The minimum objective function value of 186.00 was obtained for test problem $7 \times 7 \mathrm{~A}$ while the objective function value of the optimal discrete solution for test problem $10 \times 10 \mathrm{~A}$ was 8.00 , respectively. The results of $\mathrm{BB}$ optimization executed on test problems $7 \times 7 \mathrm{~B}$ and $10 \times 10 \mathrm{~B}$ are separately shown in Tables 3 and 4 . 
Table 1. Optimal discrete solution for test problem $7 \times 7 \mathrm{~A}$

Discrete transporting flow $x_{i, j}$

\begin{tabular}{ccccccc}
\hline 19 & 1 & 1 & 1 & 1 & 3 & 1 \\
\hline 0 & 19 & 5 & 1 & 1 & 1 & 1 \\
\hline 1 & 0 & 14 & 1 & 1 & 1 & 7 \\
\hline 0 & 0 & 0 & 18 & 1 & 1 & 0 \\
\hline 0 & 0 & 0 & 0 & 20 & 0 & 0 \\
\hline 0 & 0 & 0 & 1 & 1 & 18 & 0 \\
\hline 0 & 0 & 0 & 1 & 1 & 1 & 17 \\
\hline
\end{tabular}

Objective function: 186.00

Table 2. Optimal discrete solution for test problem $10 \times 10 \mathrm{~A}$

Discrete transporting flow $x_{i, j}$

\begin{tabular}{cccccccccc}
\hline 4 & 0 & 4 & 0 & 0 & 0 & 0 & 0 & 0 & 0 \\
\hline 0 & 0 & 2 & 0 & 3 & 0 & 0 & 3 & 0 & 0 \\
\hline 0 & 0 & 2 & 0 & 0 & 0 & 0 & 0 & 0 & 0 \\
\hline 4 & 2 & 4 & 4 & 4 & 4 & 2 & 0 & 2 & 0 \\
\hline 3 & 0 & 9 & 0 & 0 & 0 & 0 & 0 & 0 & 0 \\
\hline 0 & 0 & 1 & 0 & 0 & 0 & 0 & 0 & 0 & 0 \\
\hline 0 & 0 & 2 & 0 & 0 & 0 & 0 & 4 & 0 & 0 \\
\hline 4 & 0 & 4 & 1 & 4 & 1 & 0 & 4 & 0 & 0 \\
\hline 4 & 0 & 4 & 0 & 0 & 6 & 0 & 3 & 0 & 1 \\
\hline 0 & 0 & 1 & 0 & 0 & 0 & 0 & 0 & 0 & 0 \\
\hline & & & & & Objective function: 8.00 \\
\hline
\end{tabular}

Table 3. Optimal discrete solution for test problem $7 \times 7 \mathrm{~B}$

\begin{tabular}{ccccccc}
\hline \multicolumn{7}{c}{ Discrete transporting flow $x_{i, j}$} \\
\hline 19 & 0 & 0 & 4 & 0 & 4 & 0 \\
\hline 1 & 19 & 0 & 0 & 4 & 0 & 4 \\
\hline 0 & 1 & 20 & 0 & 0 & 0 & 4 \\
\hline 0 & 0 & 0 & 19 & 1 & 0 & 0 \\
\hline 0 & 0 & 0 & 0 & 20 & 0 & 0 \\
\hline 0 & 0 & 0 & 0 & 0 & 20 & 0 \\
\hline 0 & 0 & 0 & 0 & 1 & 1 & 18 \\
\hline \multicolumn{7}{c}{} \\
\hline 0
\end{tabular}

Table 4. Optimal discrete solution for test problem $10 \times 10 \mathrm{~B}$

\begin{tabular}{cccccccccc}
\hline \multicolumn{10}{c}{ Discrete transporting flow $x_{i, j}$} \\
\hline 0 & 2 & 1 & 4 & 1 & 0 & 0 & 0 & 0 & 0 \\
\hline 0 & 0 & 0 & 0 & 0 & 0 & 0 & 8 & 0 & 0 \\
\hline 0 & 0 & 0 & 0 & 0 & 0 & 2 & 0 & 0 & 0 \\
\hline 11 & 0 & 10 & 1 & 0 & 3 & 0 & 0 & 0 & 1 \\
\hline 0 & 0 & 10 & 0 & 0 & 0 & 0 & 0 & 2 & 0 \\
\hline 0 & 0 & 1 & 0 & 0 & 0 & 0 & 0 & 0 & 0 \\
\hline 0 & 0 & 0 & 0 & 0 & 0 & 0 & 6 & 0 & 0 \\
\hline 8 & 0 & 10 & 0 & 0 & 0 & 0 & 0 & 0 & 0 \\
\hline 0 & 0 & 0 & 0 & 10 & 8 & 0 & 0 & 0 & 0 \\
\hline 0 & 0 & 1 & 0 & 0 & 0 & 0 & 0 & 0 & 0 \\
\hline
\end{tabular}

Objective function: 147.00
For test problem $7 \times 7 \mathrm{~B}$, the attained minimum value of the objective function was 350.00 . The optimal discrete solution of test problem $10 \times 10 \mathrm{~B}$ contains objective function value of 147.00 . In following tests, the $\mathrm{BB}$ optimization method was employed to solve $7 \times 7 \mathrm{C}$ and $10 \times 10 \mathrm{C}$ problems. After executed optimization, the $\mathrm{BB}$ algorithm reported optimal discrete solutions for both aforesaid problems as presented in Tables 5 and 6.

The achieved optimal discrete solution for test problem $7 \times 7 \mathrm{C}$ indicates the minimum objective function value of 2648.00 while the one for $10 \times 10 \mathrm{C}$ problem shows the minimum value of 4466.00 . In dealing with test problems $7 \times 7 \mathrm{D}$ and $10 \times 10 \mathrm{D}$, the convergence of the $\mathrm{BB}$ algorithm was achieved at optimal discrete solutions demonstrated individually in Tables 7 and 8 .

Table 7 points out the found optimal discrete solution for test problem $7 \times 7 \mathrm{D}$ and its minimum objective function value of 480.16 while Table 8 shows the one attained for the problem $10 \times 10 \mathrm{D}$ which indicates the minimum objective function value of 377.25 . The optimal discrete solutions of test problems $7 \times 7 \mathrm{E}$ and $10 \times 10 \mathrm{E}$ reached by $\mathrm{BB}$ approach are given in Tables 9 and 10 , respectively.

In view of Table 9, one can see that the optimal discrete solution for test problem $7 \times 7 \mathrm{E}$ was achieved at the minimum objective function value of 556.19. For test problem $10 \times 10 \mathrm{E}$, Table 10 highlights that the minimum of the objective function was found at value of 71.72.

Table 5. Optimal discrete solution for test problem $7 \times 7 \mathrm{C}$

\begin{tabular}{ccccccc}
\hline \multicolumn{7}{c}{ Discrete transporting flow $x_{i, j}$} \\
\hline 20 & 0 & 1 & 2 & 2 & 2 & 0 \\
\hline 0 & 20 & 2 & 2 & 2 & 0 & 2 \\
\hline 0 & 0 & 17 & 1 & 1 & 2 & 4 \\
\hline 0 & 0 & 0 & 18 & 1 & 0 & 1 \\
\hline 0 & 0 & 0 & 0 & 20 & 0 & 0 \\
\hline 0 & 0 & 0 & 0 & 0 & 20 & 0 \\
\hline 0 & 0 & 0 & 0 & 0 & 1 & 19 \\
\hline & & & & Objective function: 2648.00 \\
\hline
\end{tabular}

Table 6. Optimal discrete solution for test problem $10 \times 10 \mathrm{C}$

\begin{tabular}{cccccccccc}
\hline \multicolumn{10}{c}{ Discrete transporting flow $x_{i, j}$} \\
\hline 1 & 0 & 3 & 0 & 2 & 0 & 0 & 2 & 0 & 0 \\
\hline 1 & 0 & 2 & 0 & 2 & 0 & 0 & 2 & 1 & 0 \\
\hline 0 & 0 & 2 & 0 & 0 & 0 & 0 & 0 & 0 & 0 \\
\hline 4 & 2 & 4 & 5 & 2 & 3 & 2 & 2 & 1 & 1 \\
\hline 0 & 0 & 8 & 0 & 1 & 0 & 0 & 3 & 0 & 0 \\
\hline 0 & 0 & 1 & 0 & 0 & 0 & 0 & 0 & 0 & 0 \\
\hline 0 & 0 & 4 & 0 & 0 & 0 & 0 & 2 & 0 & 0 \\
\hline 11 & 0 & 5 & 0 & 1 & 0 & 0 & 1 & 0 & 0 \\
\hline 2 & 0 & 3 & 0 & 3 & 8 & 0 & 2 & 0 & 0 \\
\hline 0 & 0 & 1 & 0 & 0 & 0 & 0 & 0 & 0 & 0 \\
\hline
\end{tabular}

Objective function: 4466.00 
Table 7. Optimal discrete solution for test problem $7 \times 7 \mathrm{D}$

\begin{tabular}{ccccccc}
\hline \multicolumn{7}{c}{ Discrete transporting flow $x_{i, j}$} \\
\hline 20 & 7 & 0 & 0 & 0 & 0 & 0 \\
\hline 0 & 13 & 15 & 0 & 0 & 0 & 0 \\
\hline 0 & 0 & 5 & 0 & 0 & 0 & 20 \\
\hline 0 & 0 & 0 & 20 & 0 & 0 & 0 \\
\hline 0 & 0 & 0 & 0 & 20 & 0 & 0 \\
\hline 0 & 0 & 0 & 0 & 0 & 20 & 0 \\
\hline 0 & 0 & 0 & 3 & 6 & 5 & 6 \\
\hline
\end{tabular}

Objective function: 480.16

Table 8. Optimal discrete solution for test problem $10 \times 10 \mathrm{D}$

Discrete transporting flow $x_{i, j}$

\begin{tabular}{cccccccccc}
\hline 1 & 2 & 0 & 5 & 0 & 0 & 0 & 0 & 0 & 0 \\
\hline 0 & 0 & 0 & 0 & 0 & 0 & 0 & 8 & 0 & 0 \\
\hline 0 & 0 & 0 & 0 & 0 & 0 & 2 & 0 & 0 & 0 \\
\hline 0 & 0 & 22 & 0 & 0 & 3 & 0 & 0 & 0 & 1 \\
\hline 0 & 0 & 10 & 0 & 0 & 0 & 0 & 0 & 2 & 0 \\
\hline 0 & 0 & 0 & 0 & 0 & 1 & 0 & 0 & 0 & 0 \\
\hline 0 & 0 & 0 & 0 & 0 & 0 & 0 & 6 & 0 & 0 \\
\hline 18 & 0 & 0 & 0 & 0 & 0 & 0 & 0 & 0 & 0 \\
\hline 0 & 0 & 0 & 0 & 11 & 7 & 0 & 0 & 0 & 0 \\
\hline 0 & 0 & 1 & 0 & 0 & 0 & 0 & 0 & 0 & 0 \\
\hline & & & & & Objective function: 377.25
\end{tabular}

Table 9. Optimal discrete solution for test problem $7 \times 7 \mathrm{E}$

\begin{tabular}{ccccccc}
\hline \multicolumn{7}{c}{ Discrete transporting flow $x_{i, j}$} \\
\hline 0 & 1 & 0 & 0 & 0 & 0 & 26 \\
\hline 0 & 1 & 0 & 0 & 14 & 13 & 0 \\
\hline 0 & 0 & 0 & 13 & 12 & 0 & 0 \\
\hline 0 & 8 & 0 & 0 & 0 & 12 & 0 \\
\hline 0 & 0 & 20 & 0 & 0 & 0 & 0 \\
\hline 0 & 10 & 0 & 10 & 0 & 0 & 0 \\
\hline 20 & 0 & 0 & 0 & 0 & 0 & 0 \\
\hline \multicolumn{7}{c}{ Objective function: 556.19} \\
\hline
\end{tabular}

Table 10. Optimal discrete solution for test problem $10 \times 10 \mathrm{E}$

\begin{tabular}{cccccccccc}
\hline \multicolumn{10}{c}{ Discrete transporting flow $x_{i, j}$} \\
\hline 0 & 2 & 0 & 2 & 2 & 0 & 0 & 2 & 0 & 0 \\
\hline 0 & 0 & 1 & 0 & 3 & 0 & 0 & 2 & 2 & 0 \\
\hline 0 & 0 & 0 & 1 & 0 & 0 & 0 & 1 & 0 & 0 \\
\hline 0 & 0 & 26 & 0 & 0 & 0 & 0 & 0 & 0 & 0 \\
\hline 0 & 0 & 4 & 0 & 1 & 0 & 2 & 4 & 0 & 1 \\
\hline 0 & 0 & 0 & 0 & 1 & 0 & 0 & 0 & 0 & 0 \\
\hline 0 & 0 & 1 & 2 & 0 & 0 & 0 & 3 & 0 & 0 \\
\hline 18 & 0 & 0 & 0 & 0 & 0 & 0 & 0 & 0 & 0 \\
\hline 1 & 0 & 1 & 0 & 3 & 11 & 0 & 2 & 0 & 0 \\
\hline 0 & 0 & 0 & 0 & 1 & 0 & 0 & 0 & 0 & 0 \\
\hline
\end{tabular}

Objective function: 71.72
Table 11. Optimal discrete solution for test problem $7 \times 7 \mathrm{~F}$ Discrete transporting flow $x_{i, j}$

\begin{tabular}{ccccccc}
\hline 6 & 9 & 0 & 0 & 0 & 0 & 12 \\
\hline 2 & 0 & 2 & 0 & 0 & 12 & 12 \\
\hline 0 & 9 & 16 & 0 & 0 & 0 & 0 \\
\hline 0 & 0 & 0 & 11 & 9 & 0 & 0 \\
\hline 0 & 0 & 0 & 12 & 8 & 0 & 0 \\
\hline 0 & 0 & 0 & 0 & 9 & 11 & 0 \\
\hline 12 & 2 & 2 & 0 & 0 & 2 & 2 \\
\hline \multicolumn{5}{c}{ Objective function: 162.25} \\
\hline
\end{tabular}

Table 12. Optimal discrete solution for test problem $10 \times 10 \mathrm{~F}$ Discrete transporting flow $x_{i, j}$

\begin{tabular}{rrrrrrrrrr}
\hline 0 & 2 & 6 & 0 & 0 & 0 & 0 & 0 & 0 & 0 \\
\hline 1 & 0 & 0 & 0 & 5 & 0 & 0 & 0 & 2 & 0 \\
\hline 0 & 0 & 0 & 0 & 0 & 0 & 2 & 0 & 0 & 0 \\
\hline 0 & 0 & 14 & 5 & 6 & 0 & 0 & 0 & 0 & 1 \\
\hline 6 & 0 & 6 & 0 & 0 & 0 & 0 & 0 & 0 & 0 \\
\hline 0 & 0 & 0 & 0 & 0 & 1 & 0 & 0 & 0 & 0 \\
\hline 6 & 0 & 0 & 0 & 0 & 0 & 0 & 0 & 0 & 0 \\
\hline 6 & 0 & 6 & 0 & 0 & 6 & 0 & 0 & 0 & 0 \\
\hline 0 & 0 & 0 & 0 & 0 & 4 & 0 & 14 & 0 & 0 \\
\hline 0 & 0 & 1 & 0 & 0 & 0 & 0 & 0 & 0 & 0 \\
\hline & & & & & & Objective function: 127.98
\end{tabular}

The lattermost experiments were done on $7 \times 7 \mathrm{~F}$ and $10 \times 10 \mathrm{~F}$ problems where the $\mathrm{BB}$ search resulted in optimal solutions as presented in Tables 11 and 12, respectively. The obtained optimal discrete solution of test problem $7 \times 7 \mathrm{~F}$ shows the minimum objective function value of 162.25 . Considering the $10 \times 10 \mathrm{~F}$ test problem, the discrete optimality of its solution was achieved as soon as the objective function indicated the minimum value of 127.98 .

It should be pointed out that, the total solver times required by the $\mathrm{BB}$ algorithm to find optimal discrete solutions for test problems from defined starting points were in most cases less than a second. The longest process time of about five minutes was exceptionally needed for BB algorithm to obtain the optimal solution for test problem $7 \times 7 \mathrm{~F}$ which included the most scattered superstructure of alternatives for cost function values.

\section{Discussion}

The following section discusses results of executed experiments and modelling capabilities of both applied optimization techniques. For this purpose, Table 13 presents a comparison between results obtained here by the BB based MILP optimization applied on the linearized NDTP model and previously reported results (Klanšek 2014) gained employing five different MINLP algorithms on the original NDTP model. 
Table 13. Comparison of optimization results for test problems

\begin{tabular}{ccccccc}
\hline Test problem & CPLEX & AlphaECP & BARON & DICOPT & LINDOGlobal & SBB \\
\hline $7 \times 7 \mathrm{~A}$ & 186.00 & 171.59 & - & 190.89 & 322.87 & 244.13 \\
\hline $7 \times 7 \mathrm{~B}$ & 350.00 & 362.96 & - & 350.97 & 397.46 & 349.98 \\
\hline $7 \times 7 \mathrm{C}$ & 2648.00 & 2648.00 & 2648.00 & 2648.00 & 2648.00 & 2648.00 \\
\hline $7 \times 7 \mathrm{D}$ & 480.16 & 556.21 & 480.16 & 601.78 & 480.16 & 557.81 \\
\hline $7 \times 7 \mathrm{E}$ & 556.19 & 1020.12 & 598.42 & 1083.68 & 1099.15 & 861.45 \\
\hline $7 \times 7 \mathrm{~F}$ & 162.25 & 1009.95 & - & 610.89 & 900.58 & 444.58 \\
\hline $10 \times 10 \mathrm{~A}$ & 8.00 & 66.89 & - & 92.38 & 86.86 & 91.37 \\
\hline $10 \times 10 \mathrm{~B}$ & 147.00 & 176.59 & - & 176.59 & 177.99 & 169.59 \\
\hline $10 \times 10 \mathrm{C}$ & 4466.00 & 4530.00 & 4538.00 & 5123.00 & 4758.00 & 4466.00 \\
\hline $10 \times 10 \mathrm{D}$ & 377.25 & 404.18 & 377.25 & 443.55 & 487.58 & 427.50 \\
\hline $10 \times 10 \mathrm{E}$ & 71.72 & 81.45 & 71.79 & 80.47 & 80.33 & 73.73 \\
\hline $10 \times 10 \mathrm{~F}$ & 127.98 & 768.78 & - & 315.44 & 884.01 & 246.24 \\
\hline
\end{tabular}

Notes: For more details about MINLP optimization results found by AlphaECP, BARON, DICOPT, LINDOGlobal and SBB, see reference (Klanšek 2014).

The results of MILP optimization were found here by the $\mathrm{BB}$ method that operates within the CPLEX software while the reference MINLP results from the previous research were attained by the ECP method of the AlphaECP software, the BR method of the BARON software, the AP/OA/ER method of the DICOPT software, the $\mathrm{BC}$ method of the LINDOGlobal software, and the SBB method of the same designated software.

On the basis of comparison between achieved results, it was established, that the BB based MILP optimization employed on linearized NDTP model, with the exception of very few cases which are discussed in continuation of this paper, obtained smaller or equal objective function values for test problems than the considered MINLP methods applied on the same test problems using original NDTP model.

By way of illustration, Table 13 demonstrates that CPLEX found higher objective function values than AlphaECP for test problem $7 \times 7 \mathrm{~A}$ and SBB for test problem $7 \times 7 \mathrm{~B}$. The main cause for such results arose from the fact that cost function values in MINLP models of test problems $7 \times 7 \mathrm{~A}$ and $7 \times 7 \mathrm{~B}$ were generated using continuous nonlinear approximations for piece-wise linear functions, i.e. expressions $A\left(x_{i, j}\right)$ and $B\left(x_{i, j}\right)$ given in Appendix A.2, while those in MILP models for the same test problems were determined by exact discrete values, as presented in Figs 1 and 2, respectively.

For test problem $7 \times 7 \mathrm{~B}$, it was ascertained that differences between approximated cost function values and the exact ones had nearly negligible impact on the quality of solutions, mostly because of high values of curvefitting parameter $P B$. On the other hand, in the case of test problem $7 \times 7 \mathrm{~A}$, the mentioned differences had a certain effect on the optimal objective function value.

More precisely, on each skip from one step to next, for example when transporting flow $x_{i, j}$ is equal $2,4,6,8$ and 10, the five-step function $A\left(x_{i, j}\right)$ gives approximated cost values $0.5,1.5,2.5,3.5$ and 4.5 instead of accurate ones, i.e. 1, 2, 3, 4 and 5, respectively, see Appendix A.2 and Fig. 1. Furthermore, that feature of expression $A\left(x_{i, j}\right)$ cannot be reduced by sharpening the function with possibly higher values of curve fitting parameter $P A$. Since the obtained MINLP solution of test problem $7 \times 7 \mathrm{~A}$ comprehended some transporting flows $x_{i, j}$ with values 2 and 6 (Klanšek 2014), the objective function value reported by AlphaECP optimization algorithm was accordingly underestimated.

Based on the experience acquired during modelling and solution process as well as gained results of tests, the advantages and the drawbacks of MILP and MINLP models for solving the NDTP are summarized in Table 14.

Table 14. Comparison of MILP and MINLP models for NDTP

\begin{tabular}{lcl}
\hline $\begin{array}{c}\text { Characteristics/effectiveness } \\
\text { measures }\end{array}$ & MILP model & MINLP model \\
\hline Result quality & Higher & Lower \\
\hline Result accuracy & Same & Same \\
\hline Solution time & Lower & Higher \\
\hline Modelling capabilities & Lower & Higher \\
\hline Modelling effort & Higher & Lower \\
\hline
\end{tabular}

The executed tests indicated that the $\mathrm{BB}$ based MILP optimization employed on suitably linearized NDTP model may sometimes give exceedingly more effective solution in comparison with the MINLP optimization applied on the original NDTP model, especially in cases when the objective function is nonconvex. However, when the superstructure of alternatives for cost function values is combinatorial, highly scattered or follows very nonconvex pattern, the BB search process for the optimal solution of linearized NDTP may require additional solution time.

In this respect, the results of test problems $7 \times 7 \mathrm{~F}$ and $10 \times 10 \mathrm{~F}$ found by MILP optimization show that the number of binary variables had larger impact on the BB 
process time than the number of continuous variables and constraints. Considering the accuracy of the results, both optimization techniques are expected to obtain exact solution for the NDTP. Although the results of performed tests demonstrated better solution efficiency in favour of MILP approach, the modelling capabilities can be identified as the advantage of the MINLP optimization.

Namely, the MINLP model of the NDTP may contain a wide variety of nonlinear functions. The MINLP models are thus especially applicable for those transportation problems where a large number of discrete variables of the same type follow the same nonlinear pattern. In such cases, the nonlinear approximation functions may be used in the NDTP model for easier dealing with a large-sized superstructure of discrete alternatives.

\section{Conclusions}

The NDTP problem appears in real operations when single merchandise, that can be counted in number of pieces (such as cargo in containers, goods on wooden pallets, semi-finished or finished metallurgical products, etc.), should be cost effectively transported from a number of suppliers to a number of demanders taking into account the feature of economy of scale where the unit cost of transporting cargo decreases nonlinearly as the amount of cargo increases. For example, the economy of scale may involve the unit cost reduction originated out of the massification of transportation as well as larger modes (such as megaships), distribution centres and terminals.

The aim of this paper was to demonstrate a comparison between MILP and MINLP approaches to exact optimal solution of the NDTP. The paper discussed the results of experiments obtained applying both techniques on a set of reference test problems as well as their modelling capabilities. The set of test problems included the $7 \times 7$ and the $10 \times 10$ node problems with objective functions that contained cost values generated on basis of six different discretized nonlinear expressions.

The results of performed experiments demonstrated that the MILP optimization applied on appropriately linearized NDTP model can obtain more effective solution when compared with the MINLP optimization employed on the original NDTP model. This study demonstrated that the MILP approach holds advantages over the MINLP technique in the field of solving transportation problems from the viewpoint of solution time and result quality while it requires higher effort for dealing with the input data on account of lower modelling capabilities. Thus, the MILP approach may be suitably used for small- and medium-sized transportation problems with reasonable amount of input data to be filled within the optimization model or in cases when large-size input data can be handled by data sets.

Such outcome of executed tests was also anticipated to some extent, since the field of MINLP optimization is significantly more complex and has not yet reached the state of maturity and reliability as MILP optimiza- tion. Irrespective of the fact that the results of performed tests demonstrated better solution efficiency in favour of MILP approach, it can be established that the MINLP optimization can, in many cases, found acceptable exact solutions for different NDTPs, especially for those with convex objective functions.

In this sense, the modelling capabilities represent the main advantage of MINLP approach that comes most to the fore in cases when a large-sized superstructure of discrete alternatives can be more efficiently handled within the NDTP model using nonlinear terms. Finally, the application of nonlinear functions may also enable more compact NDTP model formulation as well as acceleration of model management tasks, such as transforming the data into model parameters and modifying the model.

\section{References}

Ağralı, S.; Geunes, J.; Taşkın, Z. C. 2012. A facility location model with safety stock costs: analysis of the cost of single-sourcing requirements, Journal of Global Optimization 54(3): 551-581.

http://dx.doi.org/10.1007/s10898-011-9777-z

Brooke, A.; Kendrick, D.; Meeraus, A.; Raman, R. 2012. GAMS - A User's Guide: Monograph. GAMS Development Corporation, Washington. $316 \mathrm{p}$.

Carrizosa, E.; Ushakov, A.; Vasilyev, I. 2012. A computational study of a nonlinear minsum facility location problem, Computers \& Operations Research 39(11): 2625-2633. http://dx.doi.org/10.1016/j.cor.2012.01.009

Christensen, T. R. L.; Andersen, K. A.; Klose, A. 2013. Solving the single-sink, fixed-charge, multiple-choice transportation problem by dynamic programming, Transportation Science 47(3): 428-438.

http://dx.doi.org/10.1287/trsc.1120.0431

Dangalchev, C. A. 1996. Partially-linear transportation problems, European Journal of Operational Research 91(3): 623-633. http://dx.doi.org/10.1016/0377-2217(94)00367-X

GAMS/CPLEX 12.0 User Notes. 2012. ILOG Inc., 94-142. Available from Internet: http://www.gams.com/dd/docs/ solvers/cplex.pdf

Kameshwaran, S.; Narahari, Y. 2009. Nonconvex piecewise linear knapsack problems, European Journal of Operational Research 192(1): 56-68. http://dx.doi.org/10.1016/j.ejor.2007.08.044

Klanšek, U. 2014. Solving the nonlinear discrete transportation problem by MINLP optimization, Transport 29(1): 1-11. http://dx.doi.org/10.3846/16484142.2013.815134

Land, A. H.; Doig, A. G. 1960. An automatic method of solving discrete programming problems, Econometrica 28(3): 497-520. http://dx.doi.org/10.2307/1910129

Leyffer, S. 2001. Integrating SQP and branch-and-bound for mixed integer nonlinear programming, Computational Optimization and Applications 18(3): 295-309. http://dx.doi.org/10.1023/A:1011241421041

Lin, Y.; Schrage, L. 2009. The global solver in the LINDO API, Optimization Methods and Software 24(4-5): 657-668. http://dx.doi.org/10.1080/10556780902753221

Madadi, A.; Kurz, M. E.; Ashayeri, J. 2010. Multi-level inventory management decisions with transportation cost consideration, Transportation Research Part E: Logistics and Transportation Review 46(5): 719-734. http://dx.doi.org/10.1016/j.tre.2009.12.012 
Michalewicz, Z.; Vignaux, G. A.; Hobbs, M. 1991. A nonstandard genetic algorithm for the nonlinear transportation problem, ORSA Journal on Computing 3(4): 307-316. http://dx.doi.org/10.1287/ijoc.3.4.307

Mizutani, T.; Yamashita, M. 2013. Correlative sparsity structures and semidefinite relaxations for concave cost transportation problems with change of variables, Journal of Global Optimization 56(3): 1073-1100.

http://dx.doi.org/10.1007/s10898-012-9924-1

Monteiro, M. M.; Leal, J. E.; Raupp, F. M. P. 2010. A four-type decision-variable MINLP model for a supply chain network design, Mathematical Problems in Engineering 2010: 1-16. http://dx.doi.org/10.1155/2010/450612

Ozsen, L.; Daskin, M. S.; Coullard, C. R. 2009. Facility location modeling and inventory management with multisourcing, Transportation Science 43(4): 455-472.

http://dx.doi.org/10.1287/trsc.1090.0268

Romeijn, H. E.; Sargut, F. Z. 2011. The stochastic transportation problem with single sourcing, European Journal of Operational Research 214(2): 262-272.

http://dx.doi.org/10.1016/j.ejor.2011.04.040

Ryoo, H. S.; Sahinidis, N. V. 1996. A branch-and-reduce approach to global optimization, Journal of Global Optimization 8(2): 107-138. http://dx.doi.org/10.1007/BF00138689

\section{APPENDIX}

\section{A.1. Cost matrices and source/destination capacities}

$7 \times 7$ cost matrix and source/destination capacities

\begin{tabular}{lccccccc}
\hline Source $s_{i}:$ & 27 & 28 & 25 & 20 & 20 & 20 & 20 \\
\hline Destination $d_{j}:$ & 20 & 20 & 20 & 23 & 26 & 25 & 26 \\
\hline Cost $c_{i, j}:$ & 0 & 21 & 50 & 62 & 93 & 77 & 1000 \\
\hline & 21 & 0 & 17 & 54 & 67 & 1000 & 48 \\
\cline { 2 - 7 } & 50 & 17 & 0 & 60 & 98 & 67 & 25 \\
\cline { 2 - 7 } & 62 & 54 & 60 & 0 & 27 & 1000 & 38 \\
\cline { 2 - 7 } & 93 & 67 & 98 & 27 & 0 & 47 & 42 \\
\cline { 2 - 7 } & 77 & 1000 & 67 & 1000 & 47 & 0 & 35 \\
\cline { 2 - 7 } & 1000 & 48 & 25 & 38 & 42 & 35 & 0 \\
\hline
\end{tabular}

$10 \times 10$ cost matrix and source/destination capacities

\begin{tabular}{lcccccccccc}
\hline Source $s_{i}:$ & 8 & 8 & 2 & 26 & 12 & 1 & 6 & 18 & 18 & 1 \\
\hline Destination $d_{j}:$ & 19 & 2 & 33 & 5 & 11 & 11 & 2 & 14 & 2 & 1 \\
\hline Cost $c_{i, j}:$ & 15 & 3 & 23 & 1 & 19 & 14 & 6 & 16 & 41 & 33 \\
\hline & 13 & 17 & 30 & 36 & 20 & 17 & 26 & 19 & 3 & 33 \\
\cline { 2 - 10 } & 37 & 17 & 30 & 5 & 48 & 27 & 8 & 25 & 36 & 21 \\
\cline { 2 - 10 } & 13 & 13 & 31 & 7 & 35 & 11 & 20 & 41 & 34 & 3 \\
\hline & 31 & 24 & 8 & 30 & 28 & 33 & 2 & 8 & 1 & 8 \\
\cline { 2 - 10 } & 32 & 36 & 12 & 9 & 18 & 1 & 44 & 49 & 11 & 11 \\
\cline { 2 - 10 } & 49 & 6 & 17 & 0 & 42 & 45 & 22 & 9 & 10 & 47 \\
\cline { 2 - 10 } & 2 & 21 & 18 & 40 & 47 & 27 & 27 & 40 & 19 & 42 \\
\cline { 2 - 10 } & 13 & 16 & 25 & 21 & 19 & 0 & 32 & 20 & 32 & 35 \\
\cline { 2 - 10 } & 23 & 42 & 2 & 0 & 9 & 30 & 5 & 29 & 31 & 29 \\
\hline
\end{tabular}

Sahinidis, N.; Tawarmalani, M. 2008. BARON, in GAMS - The Solver Manuals. GAMS Development Corporation, Washington, 19-32. Available from Internet: http://faculty.ses. wsu.edu/LaFrance/Linux-Server/GAMS-All-Solvers.pdf

Viswanathan, J.; Grossmann, I. E. 1990. A combined penalty function and outer-approximation method for MINLP optimization, Computers \& Chemical Engineering 14(7): 769-782. http://dx.doi.org/10.1016/0098-1354(90)87085-4

Westerlund, T.; Pettersson, F. 1995. An extended cutting plane method for solving convex MINLP problems, Computers and Chemical Engineering 19(Suppl. 1): 131-136. http://dx.doi.org/10.1016/0098-1354(95)87027-X

Westerlund, T.; Pörn, R. 2002. Solving pseudo-convex mixed integer optimization problems by cutting plane techniques, Optimization and Engineering 3(3): 253-280.

http://dx.doi.org/10.1023/A:1021091110342

Yan, S.; Luo, S.-C. 1999. Probabilistic local search algorithms for concave cost transportation network problems, European Journal of Operational Research 117(3): 511-521. http://dx.doi.org/10.1016/S0377-2217(98)00270-7

\section{A.2. Cost functions}

$$
\begin{aligned}
A\left(x_{i, j}\right)= & \arctan \left(P A\left(x_{i, j}-S\right)\right) / \pi+0.5+ \\
& \arctan \left(P A\left(x_{i, j}-2 S\right)\right) / \pi+0.5+ \\
& \arctan \left(P A\left(x_{i, j}-3 S\right)\right) / \pi+0.5+ \\
& \arctan \left(P A\left(x_{i, j}-4 S\right)\right) / \pi+0.5+ \\
& \arctan \left(P A\left(x_{i, j}-5 S\right)\right) / \pi+0.5 \\
B\left(x_{i, j}\right)= & \left(x_{i, j} / S\right)\left(\arctan \left(P B x_{i, j}\right) / \pi+0.5\right]+ \\
& \left(1-x_{i, j} / S\right)\left(\arctan \left(P B\left(x_{i, j}-S\right)\right) / \pi+0.5\right)+ \\
& \left(x_{i, j} / S-2\right)\left(\arctan \left(P B\left(x_{i, j}-2 S\right)\right) / \pi+0.5\right) \\
C\left(x_{i, j}\right)= & x_{i, j}{ }^{2} \\
D\left(x_{i, j}\right)= & x_{i, j}{ }^{0.5} \\
E\left(x_{i, j}\right)= & \left(1+\left(x_{i, j}-2 S\right)^{2}\right)^{-1}+ \\
& \left(1+\left(x_{i, j}-2.25 S\right)^{2}\right)^{-1}+ \\
& \left(1+\left(x_{i, j}-1.75 S\right)^{2}\right)^{-1} \\
F\left(x_{i, j}\right)= & x_{i, j}\left(\sin \left(5 \pi x_{i, j} / 4 S\right)+1\right)
\end{aligned}
$$

